\title{
The Application and Research of Embedded Mobile Database
}

\author{
Yang Shi \\ Department of Computer and Science, Yunyang Teachers' College, Shiyan 442000, China \\ 3637352288@qq.com
}

Keywords: Data synchronization technique, embedded mobile database, Androil.

\begin{abstract}
With respect to the current situation of logistics industries, the data synchronization technique in embedded mobile database design is researched. The focuses is on the establishment and maintenance of server databases, so as to solve the problems, like the date acquisition in data synchronization and data overload, data error and data loss in data transmission, etc. The disposal method is to achieve information anytime and anywhere by embedded portable devices, to simplify operation of mobile terminals with Androil APP, and to diminish errors and to enhance the safety and reliability of data by techniques of breakpoint resume, cache and synchronization.
\end{abstract}

\section{Introduction}

The rapid updating of mobile computing technology expands a new exploration space for the requirements of the database system which can only run on the server, people's requirement to the function of the mobile database system can no longer satisfy the existing [1,2]. At present, in the embedded control module which is based on UNIX or android operating system, the database system has become an essential part. Because the SCM has spread in all field of automation control equipment. Therefore whether it is the civilian infrastructure or the military facilities, the application of the embedded database research has basically universal [3,4]. Pick up the equipment patent technology, the embedded database is also on the Stellerite surge. With the construction of smart city, the intelligent logistics industry is also accompanied by the rise of electric business and rapid development.

\section{The Characteristics of Embedded Mobile Database}

In general, embedded CNC library mainly has the following characteristics [5].

The Spatial Degrees of Freedom. Embedded database uses wireless communication way to realize interaction with other equipment in the territory of embedded database.

The Diversity of The Network Environment. In the hole mobile computing space, different time and place make the networking environment have a huge difference. It shows its flexibility and diversity.

Discontinuity. Mobile computing is not long connected to the network environment.

The Limitations of Resource. The power supply of mobile devices can only last a few hours. The processing power and storage capacity of database are insufficient, and the embedded database system must solve these deficiencies, it also should improve the utilization rate of resources [6].

\section{The Architecture of Embedded Mobile}

Mobile computing system is composed of fixed nodes and mobile nodes.

Distributed database in mobile environment is considered to be a distributed system which make customer and fixed server node dynamically connect.

The result of the mobile database system is shown in figure 1, where the embedded mobile devices MC includes portable computer, PDA, etc. FH has no wireless communication interface, but it installs a database and database management system. MSS has wireless communication interface, and it supports mobile computing fixed nodes. 


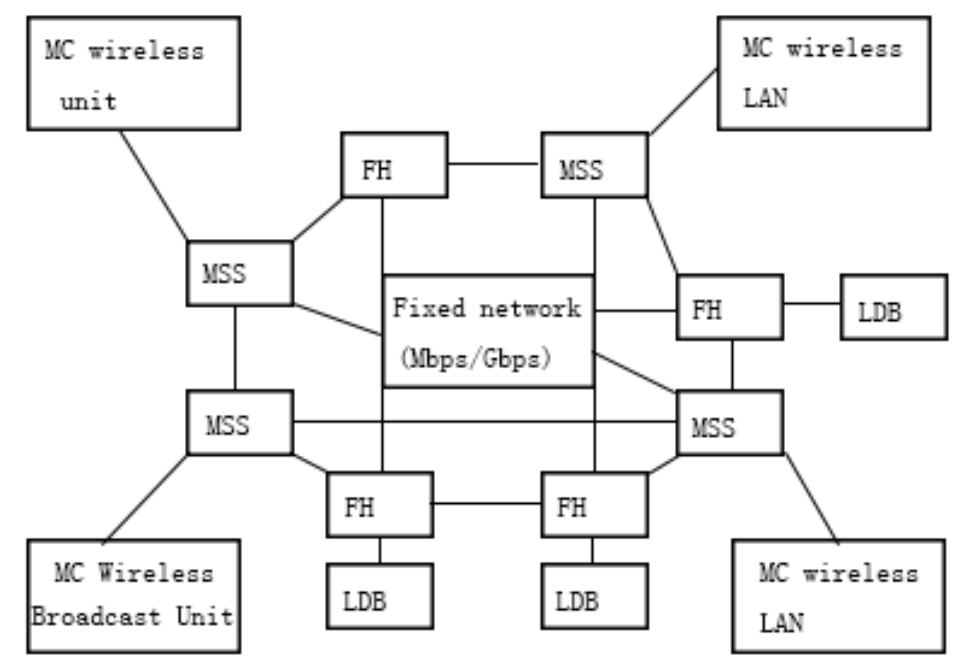

Fig. 1 The system architecture of embedded mobile databases

\section{The Design of Embedded Mobile Database Based On the Android System}

SQLite is a lightweight relational database. Because it takes up few resources, a lot of embedded devices use SQLite to store data.

SQLite is a lightweight relational database. Because it takes fewer resources, and in a lot of embedded devices SQLite is used to store data. And it now supports some mainstream operating system like like Windows/Linux/Unix, while its compatibility is very strong. We can also use a variety of development language such as c \#, Java, PHP, etc. Through the ODBC interface operations SQLite.

\section{The Design of Sqlite Database Table Acknowledgements.}

Tab 1 Logistics manager form

\begin{tabular}{cc}
\hline Field name & \multicolumn{2}{c}{ Type } \\
\hline UserID & Integer primary autoincrement \\
UserName & text \\
UserPWD & text \\
UserLevel & text \\
\hline Tab 2 The user information table \\
\hline Field name & Type \\
\hline ReceiverUser & text \\
ReceiverAddress & text \\
ReceiverUnit & text \\
ReceiverTel & text \\
ReceiverFK & text \\
\hline
\end{tabular}


Tab 3 The logistics information table

\begin{tabular}{|c|c|c|}
\hline Field name & Type & Description \\
\hline SendUser & Text & Sender \\
\hline SendAddress & Text & Send address \\
\hline SendUnit & Text & Send unit \\
\hline SendTel & Text & Sender' Tel \\
\hline SendType & Text & Items name \\
\hline SendNum & Text & Order number \\
\hline SendDate & Text & Delivery Date \\
\hline SendLog & Text & Order log \\
\hline SendCount & Integer & The number of orders \\
\hline ReceiveUser & Text & Receiver \\
\hline ReceiveAddress & Text & Receive Address \\
\hline ReceiveUnit & Text & Receive Unit \\
\hline ReceiveTel & Text & Receiver' Tel \\
\hline ReceiveSign & Text & Receiver' signature \\
\hline ReceiverSign & Text & Substitute Receiver' signature \\
\hline ReceiveDate & Text & Receive Date \\
\hline
\end{tabular}

\section{The Application of SQL Server CE Database in Warehouse Material Patrol System}

Traditionally the warehouse search is used the paper materials operations. The operating processes is that the warehouse searchers will check and fill in the records on inward and outward after receiving inventory. Then the completed form is returned to the data center, and the data center staff will manually enter the information into the computer system for processing. However, its defect is that its processing cycle is long, and the staff's workload is so big to human error after laborious and prone. According to the mobile solutions, warehouse only need to download the corresponding bom inspectors to the handheld PDA. And the optional PDA uses Windows CE operating system. Contrast to the list to check, then the result is directly recorded on a PDA on the spot and upload. A high degree of automation reduces the manual entry processing link, and after the PDA has wireless Internet function, it can extend the coverage of the system with no limit.

\section{Conclusion}

In the further, the internet of things, big data and cloud computing will become three major technologies of IT (Information Technology) industry, while data synchronization technology in embedded mobile database is a core method in the these technologies. Embedded mobile computing technology has become a research focus in the fields of database and wireless network, and has been paid much more attention. Researchers and industries pay much attention to the study of the embedded mobile database. Specially, logistics industries have got rapid development under the application of embedded mobile database technology. However, there is a lot of shortcoming in the 
design of logistics management systems used at present, which are shown in data acquisition, data transmission, data organization and data synchronization in the mobile databases.

\section{Acknowledgements}

The software engineering curriculum development based on working process oriented systematic. Project No. 2013024.

\section{References}

[1] Ding Zhiming. Key technology research of mobile databases (MS., Beijing: Graduate of Chinese Academy of Sciences, China 2002) p.28. (In Chinese)

[2] Ma Ming: Research of data consistency in Mobile Database Management, Vol. 12 (2010) No.2, p. 15-19.

[3] Liu Zhibo. Key Technology Research of Embedded Mobile Database (MS., Changchun Changchun University of Technology, China 2011) p.28. (In Chinese)

[4] Zhu Qing: Research and Implementation of Mobile database system synchronization mechanism, Vol. 22 (2004) No.5, p. 32-35.

[5] EPFL, U. Grenoble: Mobile Databases: a Report on Open Issues and Research Directions, Vol. 11 (2004) No.5, p. 48-49.

[6] Subhasish Mazumdar, Panos K. Chrysanthis: Achieving Consistency in Mobile Databases through Localization in PRO-MOTION, Vol. 7 (2002) No.4, p. 37-39. 